This is an author produced version of a paper published in Nordic Prosody: Proceedings of the XIth Conference, Tartu 2012. This paper has been peer-reviewed and is proof-corrected, but it does not include the book pagination.

Citation for the published paper: Heldner, M., Hjalmarsson, A., \& Edlund, J. (2013). Backchannel relevance spaces. In E. L. Asu \& P. Lippus (Eds.), Nordic Prosody: Proceedings of the XIth Conference, Tartu 2012 (pp. 137-146). Frankfurt am Main: Peter Lang, Germany.

\title{
Backchannel relevance spaces
}

\author{
Mattias Heldner, Anna Hjalmarsson, Jens Edlund
}

\begin{abstract}
This contribution introduces backchannel relevance spaces - intervals where it is relevant for a listener in a conversation to produce a backchannel. By annotating and comparing actual visual and vocal backchannels with potential backchannels established using a group of subjects acting as third-party listeners, we show (i) that visual only backchannels represent a substantial proportion of all backchannels; and (ii) that there are more opportunities for backchannels (i.e. potential backchannels or backchannel relevance spaces) than there are actual vocal and visual backchannels. These findings indicate that backchannel relevance spaces enable more accurate acoustic, prosodic, lexical (et cetera) descriptions of backchannel inviting cues than descriptions based on the context of actual vocal backchannels only.
\end{abstract}

\section{Introduction}

In analogy with Sacks, Schegloff, \& Jefferson's (1974) transition relevance places - places where it is relevant for another speaker to take the turn, or turntransition relevance space which is the common CA term (e.g. Schegloff, 1996), we postulate backchannel relevance spaces - intervals where it is relevant for another speaker to produce a backchannel (Yngve, 1970). Backchannels indicate that the speaker producing them is following and understanding. They are generally described as being somehow produced in the background. They are often not taken to constitute a speaking turn or to claim the floor. They may occur in the midst of another speaker's speech without disrupting that speaker. We have taken this to mean that backchannels can occur outside of turn-transition relevance spaces. This can be contrasted with the closely related concept of continuer (Schegloff, 1982). Continuers indicate an understanding that the other speaker intends to continue talking and passes the opportunity to take the turn. This can be taken to mean that continuers can only occur in positions where it would be relevant to take the turn - in turn-transition relevance spaces.

The backchannel function can be performed by short vocalisations such as 'uh huh', but descriptions of this and closely related functions (i.e. listener responses, accompaniment signals) have included the head nod as a prototypical example already from the beginning (e.g. Dittmann \& Llewellyn, 1968; Duncan, 1972; Kendon, 1967), and these observations have subsequently been replicated 
by many others (e.g. Maynard, 1987; McClave, 2000). Subsequent research has demonstrated that this function can also be conveyed via facial displays such as smiles (Brunner, 1979), brow raises (Ekman, 1979), dramatic intakes of breath and mutual gaze (e.g. Bavelas, Coates, \& Johnson, 2002), etc.

In this study, we explore the common intuition that interlocutors do not produce vocal backchannels in every backchannel relevance space (e.g. Cathcart, Carletta, \& Klein, 2003; De Kok \& Heylen, 2011; De Kok, Ozkan, Heylen, \& Morency, 2010; Huang, Morency, \& Gratch, 2010; Noguchi \& Den, 1998; Ward $\&$ Tsukahara, 2000). It has been suggested that this may be due to individual differences in speaking and listening behaviour (e.g. De Kok \& Heylen, 2011). It is also possible that some vocal backchannels may be replaced by visual backchannels such as head nods (e.g. Boholm \& Allwood, 2010; Ward \& Tsukahara, 2000; Włodarczak, Buschmeier, Malisz, Kopp, \& Wagner, 2012), or that the interlocutor passes the opportunity to produce a backchannel because it would occur too close in time to the previous one - some kind of phase restrictions on backchannels. Thus, we hypothesise that the backchannel relevance spaces are more frequent than the actual vocal and visual backchannels, or put differently, that observed vocal and visual backchannels might underestimate the number of backchannel relevance spaces. We will investigate this by annotating and comparing potential and actual backchannels in genuine two-party conversations.

Different schemes for annotating phenomena related to actual vocal and visual backchannels (e.g. communicative feedback) have been proposed (e.g. Allwood, Cerrato, Jokinen, Navarretta, \& Paggio, 2007; Bavelas, et al., 2002). Annotations of actual backchannels are labour intensive and require carefulness, but they are relatively straightforward to obtain. Potential backchannels are less so. Several different methods of identifying phenomena related to potential backchannels have been employed in the past. For example, Ward \& Tsukahara (2000) used one experienced annotator to identify appropriate places for backchannels in situations where no backchannel occurred. Several others have used techniques drawing on the wisdom-of-crowds. Noguchi \& Den (1998), and later also Huang, et al. (2010) and De Kok, et al. (2010) combined the responses of a group of subjects (e.g. $N=9$ ) asked to indicate appropriate places for backchannels by pressing a key on a keyboard while watching videos of a speaker (but not including the original listener). Similarly, Watanabe \& Yuuki (1989) asked a group of listeners to intentionally nod at appropriate places for backchannels. Huang, et al. (2010) named their technique Parasocial Consensus Sampling and De Kok, et al. (2010) called their version Parallel Listener Consensus. Furthermore, De Kok \& Heylen (2011) recorded the simultaneous vocal and visual behaviour of three designated listeners interacting with the same speaker in a video setup that gave the illusion of eye contact to all participants, while in reality 
there was only eye contact between one of the listeners and the speaker. The listeners were unaware of each other, and were led to believe that they were involved in a one-to-one interaction with the speaker. To maintain this illusion, however, the listeners were instructed to refrain from taking over the role as a speaker in any way, and only provide short feedback to the speaker. These recordings were collected in the MultiLis corpus (De Kok \& Heylen, 2011).

For the present study, we borrowed elements both from Parasocial Consensus Sampling and the MultiLis corpus setup to identify potential backchannels. In particular, we asked a group of subjects to pretend that they participated in pre-recorded conversations as active listeners by producing vocal backchannels at places they judged appropriate. An important difference to the previous studies was that our videos included both participants in two-party conversations rather than just the designated speaker. That is, the subjects were third-party observers of the full conversations, and could see and hear the original listeners.

We feel that comparing actual vocal and visual backchannels will provide interesting insights into how often backchannels are realised, and the division of labour between vocal and visual backchannels. The comparison of actual backchannels with potential backchannels (or backchannel relevance spaces) will furthermore inform us how often backchannels are omitted in genuine conversations (or alternatively, how often they could have occurred). In addition, potential backchannels will enable more accurate acoustic, prosodic, lexical (et cetera) descriptions of backchannel relevance spaces than the descriptions based on the context of actual vocal backchannels only, which has hitherto been praxis.

\section{Method}

\subsection{The Spontal corpus}

This study draws on material from the Spontal corpus (Edlund, et al., 2010). Spontal contains in excess of 60 hours of dialogue: 120 nominal half-hour sessions (the duration of each dialogue is minimally 30 minutes). There were no set tasks, and no designated speakers and listeners. The subjects were allowed to talk about anything they wanted at any point in the session. The subjects are all native speakers of Swedish. The corpus is balanced (1) as to whether the interlocutors are of the same or different gender, and (2) as to whether they know each other or not before the recording. The recordings contain high-quality audio and video, as well as motion capture data.

For the present study, we randomly chose four segments from the development set of the most recent Spontal recordings (SpontalIDs 09-20; 09-28; 09-30; 09-36), but in such a manner that they were taken from different balance groups: 
the segments included one known and one unknown same gender (male) pair, as well as one known and one unknown opposite gender pair. Each segment consisted of the first five minutes of the dialogue - that is the first five minutes following the moment when the recording assistant told the participants that the recording had started.

\subsection{Annotations}

We used ELAN (version 4.4.0, http://www.lat-mpi.eu/tools/elan/) to collect annotations of actual vocal and visual backchannels as well as potential backchannels. The interface displayed front facing videos of both participants (as in Figure 1), and audio waveforms of their individual close-talk microphone channels.

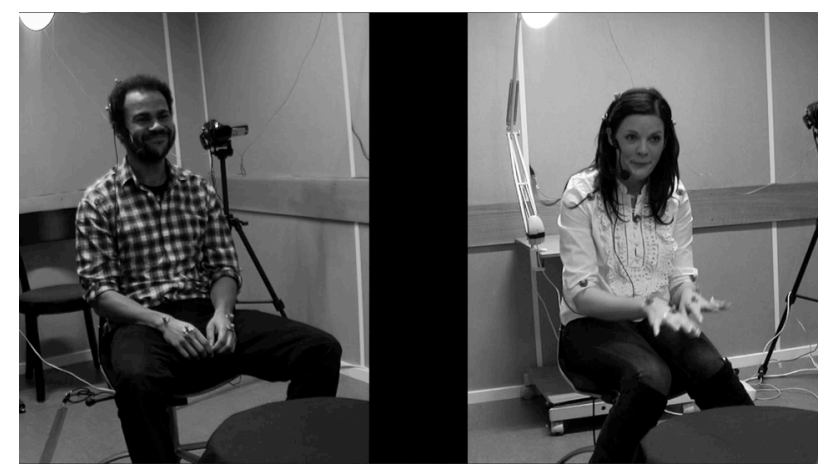

Figure 1: $\quad$ Still image from a Spontal recording session.

The annotation of actual vocal and visual backchannels was intentionally minimal and course-grained, marking only events perceived to have the communicative function of a backchannel. No distinction was made between, for example, different types of feedback, different types of head movements or different types of facial displays, as in the MUMIN framework (Allwood, et al., 2007). Nevertheless, the annotation of actual backchannels included three layers (or tiers) for each of the interlocutors:

(1)Role in conversation: One of the authors impressionistically identified intervals where participants held the roles as speakers and listeners, respectively.

(2)Actual vocal backchannels: All three authors identified short vocalisations perceived to have the communicative function of a backchannel individually, and instances where two or three annotators agreed were included in subsequent analyses.

(3)Actual visual backchannels: One of the authors identified visual gestures and facial displays perceived to have a backchannel function (including headnods, blinks, smiles, brow raises, hand gestures, et cetera), but no distinction between different types of visual backchannels will be made here. 
The annotations of vocal backchannels should be taken as more reliable than those of visual backchannels - partly because the vocal backchannels were selected by the majority votes of the annotators, and partly because it was at times difficult to determine whether a visual backchannel was present or not. The markup of role in conversation was needed to obtain estimates of backchannel frequency during listening, as there were no designated speakers and listeners in Spontal.

The potential backchannels were identified using a group of subjects $(N=7)$ producing vocal backchannels while watching the same video segments used for the annotations of actual backchannels. The subjects were instructed to pretend that they participated in the pre-recorded conversation as active listeners by producing vocal backchannels at places they judged appropriate. In addition, they were explicitly asked to look at the current speaker as much as possible. The videos were presented on a Tobii T120 eye-tracker. The sound from the videos was played over headphones. The vocal backchannels produced by the subjects were recorded using a Brüel \& Kjær 4035 headset microphone. Sound as well as gaze behaviour was recorded using the eye-tracker, although the gaze data was not analysed here.

The individual vocal backchannels were identified by means of voice activity detection using the Praat command To TextGrid Silences... using a minimum silence duration of $200 \mathrm{~ms}$ and a minimum sound duration of $150 \mathrm{~ms}$. The resulting TextGrids were imported into ELAN. Subsequently, joint silences - intervals of silence in all seven channels - were identified using the ELAN command Create annotations from gaps using the voice activity from all subjects this intermediate step gave us the complement of what we wanted. Finally, backchannel relevance spaces were identified using Create annotations from gaps again, but this time on the joint silences-tier. The resulting annotation thus captures intervals from the onset of any subject's vocal backchannel to the offset of any at least partly overlapping vocal backchannel.

\section{Results and discussion}

\subsection{Actual backchannels}

The manual annotations of actual backchannels identified 175 vocal backchannels in the material. 87 of these were accompanied by at least partly overlapping visual backchannels. In addition, 84 exclusively visual backchannels were identified giving a total of 259 actual backchannels in the material. Table 1 shows the distribution of vocal only backchannels, visual only backchannels, as well as (at least partly) overlapping vocal and visual backchannels across dialogues and 
participants (columns 3-5). Table 1 also shows the average frequencies of backchannels in listener intervals (in backchannels per minute) across dialogues and participants (columns 6-7).

Table 1: $\quad$ Distribution of vocal only backchannels, visual only backchannels, and overlapping vocal and visual backchannels across dialogues and participants, as well as average frequencies of all kinds of backchannels in listener intervals (in backchannels per minute) across dialogues and participants.

\begin{tabular}{|c|c|c|c|c|c|c|}
\hline & & & stribu & tion & Freq & uency \\
\hline & & Left & Right & Total & Left & Right \\
\hline $09-20$ & Vocal only & & 7 & 17 & & \\
\hline & Visual only & 6 & 29 & 35 & & \\
\hline & Vocal and visual & 8 & 6 & 14 & & \\
\hline & Total & 24 & 42 & 66 & 21 & 11 \\
\hline $09-28$ & Vocal only & 16 & 16 & 32 & & \\
\hline & Visual only & 14 & 1 & 15 & & \\
\hline & Vocal and visual & 11 & 13 & 24 & & \\
\hline & Total & 41 & 30 & 71 & 14 & 14 \\
\hline 09-30 & Vocal only & 13 & 9 & 22 & & \\
\hline & Visual only & 14 & 6 & 20 & & \\
\hline & Vocal and visual & 3 & 6 & 9 & & \\
\hline & Total & 30 & 21 & 51 & 11 & 9 \\
\hline $09-36$ & Vocal only & 3 & 14 & 17 & & \\
\hline & Visual only & 6 & 8 & 14 & & \\
\hline & Vocal and visual & 12 & 28 & 40 & & \\
\hline & Total & 21 & 50 & 71 & 14 & 16 \\
\hline Grand total & & & & 259 & & \\
\hline
\end{tabular}

Unsurprisingly, Table 1 shows that there is variability in the number of backchannels, as well as in the distribution of vocal and visual backchannels across dialogues. More importantly, Table 1 shows that vocal backchannels are often accompanied by at least partly overlapping visual ones, and furthermore that the visual only backchannels represent a substantial proportion (20\% to $53 \%)$ of all backchannels. Similarly, Boholm \& Allwood (2010) observed that about $26 \%$ of all instances of feedback in their material were silent, that is, visual only. Clearly, a unimodal approach, for example capturing vocal backchannels only, would result in considerably fewer actual backchannels than a multimodal approach. 
Table 1 also shows that the highest backchannel frequency (vocal plus visual) during listener intervals found in any of the dialogues is about 20 backchannels per minute, and furthermore that the average backchannel frequency is about 14 backchannels per minute when all kinds of backchannels are included. This suggests that an approximation of the maximum backchannel frequency in spoken dialogue systems acting as active listeners would be around one backchannel every three seconds.

\subsection{Potential backchannels}

Table 2 shows the results for potential backchannels in the four dialogues: there are individual counts for vocal backchannels for the seven subjects, as well as individual frequencies expressed in backchannels per minute. In addition, Table 2 includes averages across subjects, counts and frequencies for actual backchannels repeated from the previous section for simplicity, and the counts and frequencies for potential backchannels or backchannel relevance spaces.

Table 2: $\quad$ Individual counts (C) for vocal backchannels for the seven subjects (M1 to F2), as well as individual frequencies $(F)$ expressed in backchannels per minute, across dialogues. In addition, there are averages across subjects, counts and frequencies for actual backchannels, and backchannel relevance spaces.

\begin{tabular}{|l|cc|cc|cc|cc|}
\hline & \multicolumn{2}{|c|}{$09-20$} & \multicolumn{2}{|c|}{$09-28$} & $09-30$ & \multicolumn{2}{|c|}{$09-36$} \\
\hline Subject & $C$ & $F$ & $C$ & $F$ & $C$ & $F$ & $C$ & $F$ \\
\hline M1 & 51 & 10.2 & 45 & 9 & 21 & 4.2 & 57 & 11.4 \\
M2 & 106 & 21.2 & 85 & 17 & 156 & 31.2 & 95 & 19 \\
M3 & 53 & 10.6 & 57 & 11.4 & 56 & 11.2 & 61 & 12.2 \\
M4 & 84 & 16.8 & 64 & 12.8 & 108 & 21.6 & 65 & 13 \\
M5 & 98 & 19.6 & 71 & 14.2 & 146 & 29.2 & 99 & 19.8 \\
F1 & 98 & 19.6 & 90 & 18 & 73 & 14.6 & 117 & 23.4 \\
F2 & 97 & 19.4 & 142 & 28.4 & 37 & 7.4 & 54 & 10.8 \\
\hline Average across subjects & 84 & 17 & 79 & 16 & 85 & 17 & 78 & 16 \\
\hline Actual backchannels & 66 & 13.2 & 71 & 14.2 & 51 & 10.2 & 71 & 14.2 \\
\hline Backchannel relevance spaces & 238 & 47.6 & 221 & 44.2 & 252 & 50.4 & 195 & 39 \\
\hline
\end{tabular}

Again, these results indicate that there are individual differences in backchannel frequency. Some of the subjects in the potential backchannel experiment evidently produce more backchannels than the original speakers and listeners, some less. If we ignore the individual differences and turn to the averages across sub- 
jects, we find that the group of subjects produced on average about 16 backchannels per minute, or put differently, about one backchannel every 3.7 seconds. Thus, the group of subjects produced backchannels more frequently than the original speakers and listeners (who averaged about 13 backchannels per minute or one backchannel every 4.6 seconds).

But the most striking finding here is that the number of backchannel relevance spaces is considerably higher than any individual number, including the actual backchannels. The backchannel relevance spaces are far more frequent than the actual vocal and visual backchannels. There are on average 3.5 times more backchannel relevance spaces than actual backchannels. Thus, we may safely conclude that the way we define backchannel relevance spaces, backchannel relevance spaces are considerably more frequent than the actual backchannels. The consequence of this is that actual or observed backchannels will underestimate the number places where it is relevant for a listener to produce a backchannel.

\section{Conclusions}

With the risk of stating the obvious, we still feel that it is worth stressing that the first conclusion based on the results of this study is that multimodal annotation is a necessity for studying events with the communicative function of a backchannel, at least in face-to-face conversation. Visual backchannels may co-occur with vocal backchannels, but it also appears that visual only backchannels may be used instead of vocal backchannels - that is, in the same positions as vocal backchannels. These visual only backchannels represent a substantial proportion ( $20 \%$ to $53 \%$ ) of all backchannels. Omitting visual backchannels from counts of actual backchannels will therefore underestimate the number of intervals where it is relevant for a listener to produce a backchannel.

A second conclusion to be drawn from this study is that interlocutors do not produce vocal or visual backchannels in every backchannel relevance space. The highest actual individual backchannel frequency found in any of the dialogues is about 20 backchannels per minute. Backchannel relevance spaces are considerably more frequent than the actual vocal and visual backchannels. There are on average 3.5 times more backchannel relevance spaces than actual backchannels. Counts of actual backchannels will drastically underestimate the number of intervals where it is relevant for a listener to produce a backchannel.

Descriptions of backchannel inviting cues in backchannel relevance spaces promise more accurate acoustic, prosodic, lexical (etc.) descriptions of such cues than descriptions based on the context of actual vocal backchannels only. 
In future work, we would like to explore more sophisticated means to identify backchannel relevance spaces, for example exploiting probabilities of backchannels based on the number of overlapping productions in the potential backchannel experiment. In particular, we would like to re-calculate the number of backchannel relevance spaces using probability thresholds higher than 1, which is the threshold used in the present study. We would also like to explore potential phase restrictions on backchannels. And obviously, a main goal of this work is to describe the prosody in the vicinity of backchannel relevance places to get better descriptions of backchannel inviting cues.

\section{Acknowledgements}

The research presented here was funded in part by the Swedish Research Council project Samtalets rytm/The Rhythm of Conversation (2009-1766).

\section{References}

Allwood, J., Cerrato, L., Jokinen, K., Navarretta, C., \& Paggio, P. (2007). The MUMIN coding scheme for the annotation of feedback, turn management and sequencing phenomena. Language Resources and Evaluation, 41(3), 273-287.

Bavelas, J. B., Coates, L., \& Johnson, T. (2002). Listener responses as a collaborative process: The role of gaze. Journal of Communication, 52(3), 566-580.

Boholm, M., \& Allwood, J. (2010). Repeated head movements, their function and relation to speech. In Proceedings of the Seventh conference on International Language Resources and Evaluation (LREC'10) (pp. 6-10), Valetta, Malta.

Brunner, L. J. (1979). Smiles can be back channels. Journal of Personality and Social Psychology, 37(5), 728-734.

Cathcart, N., Carletta, J., \& Klein, E. (2003). A shallow model of backchannel continuers in spoken dialogue. In Proceeding EACL '03 Proceedings of the tenth conference on European chapter of the Association for Computational Linguistics (pp. 51-58), Budapest, Hungary.

De Kok, I., \& Heylen, D. (2011). The MultiLis corpus - Dealing with individual differences in nonverbal listening behavior. In 3rd COST 2102 International Training School on Toward Autonomous, Adaptive, and Context-Aware Multimodal Interfaces: Theoretical and Practical Issues (pp. 362-375), Caserta, Italy.

De Kok, I., Ozkan, D., Heylen, D., \& Morency, L.-P. (2010). Learning and evaluating response prediction models using parallel listener consensus. In Proceedings ICMIMLMI '10 International Conference on Multimodal Interfaces and the Workshop on Machine Learning for Multimodal Interaction, Beijing, China.

Dittmann, A. T., \& Llewellyn, L. G. (1968). Relationship between vocalizations and head nods as listener responses. Journal of Personality and Social Psychology, 9(1), 79-84.

Duncan, S., Jr. (1972). Some signals and rules for taking speaking turns in conversations. Journal of Personality and Social Psychology, 23(2), 283-292. 
Edlund, J., Beskow, J., Elenius, K., Hellmer, K., Strömbergsson, S., \& House, D. (2010). Spontal: a Swedish spontaneous dialogue corpus of audio, video and motion capture. In Proceedings of the Seventh conference on International Language Resources and Evaluation (LREC'10) (pp. 2992-2995), Valetta, Malta.

Ekman, P. (1979). About brows: Emotional and conversational signals. In M. von Cranach, K. Foppa, W. Lepenies \& D. Ploog (Eds.), Human ethology (pp. 169-202), Cambrige: Cambridge University Press.

Huang, L., Morency, L.-P., \& Gratch, J. (2010). Parasocial consensus sampling: Combining multiple perspectives to learn virtual human behavior. In Proceedings of the 9th International Conference on Autonomous Agents and Multiagent Systems (AAMAS-2010) (pp. 1265-1272), Toronto, Canada.

Kendon, A. (1967). Some functions of gaze-direction in social interaction. Acta Psychologica, 26(C), 22-63.

Maynard, S. K. (1987). Interactional functions of a nonverbal sign: Head movement in japanese dyadic casual conversation. Journal of Pragmatics, 11(5), 589-606.

McClave, E. Z. (2000). Linguistic functions of head movements in the context of speech. Journal of Pragmatics, 32(7), 855-878.

Noguchi, H., \& Den, Y. (1998). Prosody-based detection of the context of backchannel responses. In Proceedings of the Fifth International Conference on Spoken Language Processing (ICSLP'98) (pp. 487-490), Sydney, Australia.

Sacks, H., Schegloff, E. A., \& Jefferson, G. (1974). A simplest systematics for the organization of turn-taking for conversation. Language, 50, 696-735.

Schegloff, E. A. (1982). Discourse as an interactional achievement: Some uses of 'uh huh' and other things that come between sentences. In D. Tannen (Ed.), Analyzing Discourse: Text and Talk (pp. 71-93), Washington, D.C., USA: Georgetown University Press.

Schegloff, E. A. (1996). Turn organization: One intersection of grammar and interaction. In E. Ochs, E. A. Schegloff \& S. A. Thompson (Eds.), Interaction and Grammar (pp. 52133), Cambridge: Cambridge University Press.

Ward, N., \& Tsukahara, W. (2000). Prosodic features which cue back-channel responses in English and Japanese. Journal of Pragmatics, 32, 1177-1207.

Watanabe, T., \& Yuuki, N. (1989). A voice reaction system with a visualized response equivalent to nodding. In Proceedings of the third international conference on humancomputer interaction (pp. 396-403), New York, NY, USA: Elsevier Science Inc.

Włodarczak, M., Buschmeier, H., Malisz, Z., Kopp, S., \& Wagner, P. (2012). Listener head gestures and verbal feedback expressions in a distraction task. In The Interdisciplinary Workshop on Feedback Behaviors in Dialog.

Yngve, V. H. (1970). On getting a word in edgewise. In Papers from the sixth regional meeting of the Chicago Linguistic Society (pp. 567-578), Chicago, IL, USA: Chicago Linguistic Society. 
